\title{
Preface and Overview
}

\section{Second International MDMA 'Ecstasy' Conference in Australasia: Monash University, Melbourne, Australia}

\section{PREFACE}

The Second International MDMA conference in Australasia was held at Monash University in Melbourne on July 18th-19th 2010. Research from over twenty universities was presented, in a program which included both oral papers and posters. The first Australasian MDMA conference was held earlier at the Brain Sciences Institute, Swinburne University, also in Melbourne. All attendees were active MDMA researchers, with the main aims of the conference to facilitate lively debate and creative discussion amongst fellow experts. Similar to the famous CIBA Foundation seminars first held in London more than 60 years ago, this Australasian meeting was modelled on previous MDMA symposia held at the Novartis Foundation in London (formerly CIBA Foundation) during 1998 and 2004. The papers from the two London meetings have been published as special MDMA issues, the first in Neuropsychobiology [1-4, and others], the second in the Journal of Psychopharmacology [5-7 and others]. The first Australian MDMA meeting has also been published as a special Neuropsychobiology issue [8-13 and others]. It is therefore with pleasure that we are able to publish the proceedings of this most recent conference as a special MDMA issue for the Open Addiction Journal. We would like to thank the Editor, Professor Joris Verster for his support, and welcome the journal's open access policy. The Instructions for Authors outlined the requirements for the extended abstract format, with submissions of up to 500 words, including one simple figure/table and a maximum of 10 references. This format provides more extensive information than does the regular abstract format, while still allowing authors to separately publish their work as full journal articles. The abstracts were reviewed by Jillian Broadbear and Andy Parrott in their role as guest editors. Finally, we would also like to thank the Faculty of Medicine at Monash University for their generous financial support, which minimised costs associated with attendance and enabled a number of international, interstate and younger researchers to attend.

\section{OVERVIEW}

A prominent theme to emerge from earlier conferences concerned the MDMA-associated deficits in memory and cognition [1,6, 11]. This has continued to be a focus for MDMA research with a number of new findings described at the present meeting. A study undertaken by Rouse and Bruno (University of Tasmania, Australia), measured recall of related and unrelated word lists in four participant subgroups: regular consumers of ecstasy only, non-user controls, cannabis users, and cannabis/ecstasy users. The ecstasy alone group showed significant recall deficits compared to the other three groups. Furthermore these deficits were primarily in the earlier word list presentations, suggesting that ecstasy/MDMA slows the rate of memory consolidation. Hadjiefthyvoulou and colleagues (Central Lancashire University, UK) developed a novel battery of real-time prospective memory tasks. Drug-free ecstasy/MDMA users were significantly impaired on many of these active memory tasks, which extended previous research showing prospective memory impairments in ecstasy users [14]. Hadjiefthyvoulou also noted significant deficits in executive processing, but showed that these two areas of cognitive deficit were largely independent. Rodgers et al. (Newcastle University, UK), undertook a large Internet study of self-rated memory skills, involving collaborators from British and Australian universities. Memory deficits were evident in the main sample, but intriguingly were not evident in a subset of users recruited from an MDMA-advice website. Nicotine dependency also had a contributory role, with cigarette smoking adding to the memory deficits reported by ecstasy users. Several of the papers at the Monash conference investigated the effects of co-administered drugs (polydrug use) and a complex range of positive, neutral, and negative contributory influences emerged. For instance, Matthews and Bruno (University of Tasmania, Australia) noted the contribution of alcohol in self-reported prospective memory problems, whereas cannabis was implicated in retrospective memory problems. Memory problems were also predicated by self-reported psychological stress in this study as well as in work by Scott and colleagues (National Drug and Alcohol Research Centre, Australia), who found that the regular use of methamphetamine and cannabis may be associated with an increased risk of mental health problems in ecstasy users

In previous conferences, the chemical composition of ecstasy tablets was often debated [2, 4]. Here at Monash, novel data involving the biochemical analysis of hair samples was presented by Scholey and colleagues (Swinburne University and collaborative universities). They reported that there was a high concordance between the presence of MDMA in hair and selfreported ecstasy usage, underscoring the reliability of self-report by users as well as the presence of MDMA in ecstasy pills. McElwee and Cogger (Turning Point Alcohol and Drug Centre, Australia) described an increase in polydrug usage among ecstasy users through analysis of 2,897 ecstasy-related ambulance call-outs in Melbourne over the ten year period from 2000 to 2009. Over this time the incidence of cases in which MDMA was solely implicated decreased from $34 \%$ to $11 \%$, while ecstasy-polydrug cases increased from 22\% to 40\%. Sindicich and colleagues (National Drug and Alcohol Research Centre, Australia) documented a reduction in preference for MDMA in Australia, along with an increase in cannabis, cocaine, amyl nitrate and methadrone use among regular ecstasy users. They suggested that a reduction in purity of ecstasy tablets in Australia is contributing to increased polydrug use. In the UK, MDMA was the most widely used recreational stimulant ten years ago, but more recently it has been 
overtaken by cocaine in powder form. Weekend cocaine users (some of whom were also ecstasy users), were compared with weekend ecstasy users in three small pilot studies by Parrott et al. (Swansea University, UK). The use of these drugs was associated with deficits in executive processing and memory ability. The main difference was in the duration of after-effects, with the ecstasy users reporting more psychobiological deficits than did cocaine users two days later.

MDMA is still a relatively under researched psychoactive drug, with many novel areas available for investigation. There are serotonergic pathways in the occipital lobes, so given the primacy of MDMA's effects upon serotonin [15]. MDMA might be expected to affect vision. Supportive data for this hypothesis were presented at the first Australasian Conference [10]. Brown and Bruno (Australian National University and University of Tasmania, Australia) presented a comprehensive review of their visual research programme. They have demonstrated that chronic MDMA usage affects lateral inhibition between visual-orientation neurones using a tilt-aftereffect illusion, although whether it also affects higher order integrative processing remains unclear. Stough et al. (Swinburne University, Australia) undertook a car driving simulator study, in which MDMA was compared with methamphetamine. Acute MDMA caused various driving deficits relative to placebo, including not keeping a safe distance, steering wandering, and others. Self-rated sleep quality was assessed in an online questionnaire study by Ogeil and colleagues (Monash University, Australia) using the Pittsburgh Sleep Quality Index. They reported that $69 \%$ of recreational ecstasy/MDMA users had clinically significant levels of sleep disturbance, whereas excessive daytime sleepiness was infrequent (15\%), suggesting that sleep quality may be a useful marker of MDMA-related harm. The literature of psychiatric functioning was reviewed by Parrott (Swansea University, UK), using diathesis-stress interactions as an explanatory model. This suggests that MDMA is a neurochemical and neurohormonal stressor, but individual susceptibility to drug-related problems depends on a range of predisposition and environmental co-factors [7, 12].

In relation to animal research, Salem and colleagues (University of Adelaide, Australia) demonstrated a contributory role of proinflammatory cytokines to the hyperthermic response evoked by MDMA in rats. In a related study, Anderson and colleagues (University of Adelaide, Australia) showed that microglia-mediated inflammatory responses were also involved in the hyperthermic response to MDMA in animals, suggesting that immune status of the organism may contribute to the variability of thermic responses. Smithies and Broadbear (Monash University, Australia) investigated binge MDMA dosing in rats trained to discriminate MDMA from d-amphetamine and saline. The contribution of serotonin 1A and 2A/C receptor subtypes to MDMA's discriminative stimulus effects was demonstrated before and after binge treatment, which acutely and selectively interfered with MDMA's discriminative cues. Broadbear and colleagues (Monash University, Australia) described interesting gender differences in a drug discrimination study [16]. They had previously noted that oxytocin and MDMA had similar interoceptive effects, suggesting that this neurohormone may be important for the positive affective properties of acute MDMA [8]. They subsequently reported that female rats demonstrated greater sensitivity with respect to the detection of MDMA-associated cues than did male rats, whereas male rats were more sensitive to the behaviour disrupting effects of drug treatment. Harper and colleagues (Victoria University of Wellington, New Zealand) presented a series of pre-clinical MDMA studies. A dopamine mechanism was proposed to explain MDMA-induced impairment in reference memory, since MDMA-related deficits in radial arm maze performance were found to resemble impairment measured following dopaminergic agonist treatment but not serotonin agonist or acetylcholine antagonist treatments. Harper and colleagues also reported on the cross-generalisation effects between amphetamine and MDMA in a drug discrimination paradigm, demonstrating that the overlap in their discriminative stimulus effects is dopaminergic in nature.

Several papers undertook broad overviews of MDMA's effects in humans. Fisk and colleagues (University of Central Lancashire, UK) developed a statistical model for identifying self-reported adverse effects related to ecstasy use in polydrug users. They concluded that adverse effects were positively related to both lifetime ecstasy consumption and concurrent ecstasy and alcohol use, and inversely related to time since last use, suggesting diminution of adverse outcomes with abstinence. Pennay (Turning Point Alcohol and Drug Centre, Australia) undertook a qualitative study of heavy ecstasy users, focusing on 'excessive or carnal' rates of use (i.e. bingeing). The problems reported by users included 'the sads' (adverse mental health), 'being scattered' (cognitive harms impacting on employment), along with financial and social problems. It was further noted that users' pursuit of pleasure needs to be acknowledged in harm reduction initiatives in order to engage recreational drug users. Bruno and colleagues (University of Tasmania, Australia) extended earlier findings regarding ecstasy dependence [9] by evaluating how the Severity of Dependence Scale could be adopted for detecting ecstasy dependence. They found that this brief instrument can be used to sensitively and specifically identify problem ecstasy use. Matthews and Bruno (University of Tasmania, Australia) also reported the coincidence of paranoid ideation and ecstasy use that was separable from cannabis and methamphetamine exposure, particularly in users who had early onset and high frequency of ecstasy use. Milani and colleagues (Thames Valley University, UK) investigated ecstasy dependence in users, noting that the extent of dependence was more clearly related to psychiatric distress than was overall drug consumption. O'Sullivan and Parrott (University of East London, UK) found that novice current ecstasy users reported positive cost-benefit ratios, whereas heavy/experienced users reported negative cost-benefit ratios (i.e. more problems than benefits). They suggested that the worsening cost-benefit ratio with repeated ecstasy/MDMA use could explain why the frequency of ecstasy use declines over time. Similarly, Harper and Hunt (Victoria University at Wellington, New Zealand) showed that rats were more sensitive to reinforcer strength following acute exposure to MDMA than following chronic exposure.

Several papers presented some rather unexpected findings. Remmelink and McGregor (University of Sydney, Australia) noted that MDMA did not enhance social interaction in adolescent rats, despite their earlier observations that it increased social interaction in adult rats [17]. Bedi and de Wit (University of Chicago and New York State Psychiatric Institute, USA) presented two papers describing the acute mood effects of MDMA administration in human laboratory studies. In the first study, $0.75 \mathrm{mg} / \mathrm{kg}$ caused a significant increase in self-rated 'loneliness', while a higher dose of $1.5 \mathrm{mg} / \mathrm{kg}$ led to significant increases in feelings of 'loving', 
'friendly' and 'playful' relative to the lower dose or placebo. In the second study, gender differences in responses to ecstasy were apparent, with females reporting a significant increase in feeling 'anxious' and significant reductions in 'elation' and 'positive affect'. These negative mood changes were not apparent in males. Females also showed greater cardiovascular reactivity than did males. Negative acute mood changes were however reported by both genders a laboratory study conducted by Stough and colleagues (Swinburne University, Australia), who found that 100mg acute MDMA did not increase positive mood, but did cause stronger negative moods than under placebo.

In conclusion, the Second International MDMA meeting in Australasia generated a wide variety of recent research presentations that well represent the breadth of the impact of MDMA/ecstasy use in the wider community. Many of the findings have confirmed and extended prior knowledge in established research areas; for example, MDMA's effects on memory and cognition in humans and animals. Other papers added important new data to address long-standing questions such as those regarding MDMA-ecstasy equivalence, polydrug usage, the role of dependence, sleep deficits, gender effects, and drug discrimination. Several studies covered novel areas or presented rather unexpected findings, as with visual aspects, contributions to hyperthermia by glial cell secretions, differences in adolescent/adult drug reactions, and negative mood changes to acute MDMA. We hope that further research into all these areas will generate novel papers for the next Australasian MDMA conference. This is planned for January 2013 and will be hosted by Victoria University of Wellington in New Zealand. We welcome ideas for symposia and early expressions of interest. Please email: Andy Parrott, Jillian Broadbear or Sue Schenk (the local organizer) Susan.Schenk@vuw.ac.nz.

\section{REFERENCES}

[1] McCann UD, Eligulashvili V, Ricaurte GA. (+-) 3, 4-methylenedioxy-methamphetamine ('Ecstasy')-induced serotonin neurotoxicity: clinical studies. Neuropsychobiology 2000; 42: 11-16.

[2] Schifano F. Potential human neurotoxicity of MDMA ('Ecstasy') subjective self-reports, evidence form an Italian drug addiction centre and clinical case studies. Neuropsychobiology 2000; 42: 25-33.

[3] Parrott AC. Human research on MDMA (3, 4-methylenedioxymethamphetamine) neurotoxicity: cognitive and behavioural indices of change. Neuropsychobiology 2000; 42: 17-24.

[4] Curran V. Is MDMA ('Ecstasy') neurotoxic in humans? An overview of evidence and methodological problems in research. Neuropsychobiology 2000; 42: $34-41$.

[5] Parrott AC, Marsden C. MDMA (3, 4-methylenedioxymethamphetamine) or Ecstasy: the contemporary human and animal research perspective. J Psychopharmacol 2006; 20: 143-6.

[6] Quednow BB, Jessen F, Kuhn KU, Maier W, Daum I, Wagner M. Memory deficits in abstinent MDMA (ecstasy) users: neuropsychological evidence of frontal dysfunction. J Psychopharmacol 2006; 20: 373-84.

[7] Parrott AC. MDMA in humans: factors which affect the neuropsychobiological profiles of recreational Ecstasy users, the integrative role of bio-energetic stress. J Psychopharmacol 2006; 20: 147-63.

[8] Beringer K, Tunstall B, Broadbear JH. Oxytocin as a mediator of the unique interoceptive effects of 3, 4-methylenedioxymethamphetamine (MDMA, "ecstasy") in the rat. Neuropsychobiology 2009; 60: 217.

[9] Bruno R, Mathews AJ, Topp L, Degenhardt L, Gomez R, Dunn M. Can the severity of dependence scale be usefully applied to 'Ecstasy'? Neuropsychobiology 2009; 60: 137-47.

[10] Dickson C, Bruno R, Brown J. Investigating the role of serotonin in visual orientation processing using an ecstasy (MDMA)-based research model. Neuropsychobiology 2009; 60: 204-212.

[11] Murphy PN, Wareing M, Fisk JE, Montgomery C. Executive working memory deficits in abstinent Ecstasy/MDMA users: a critical review. Neuropsychobiology 2009; 60: 159-75

[12] Parrott AC. Cortisol and MDMA (3, 4-methylenedioxymethamphetamine): neurohormonal aspects of recreational Ecstasy. Neuropsychobiology 2009; 60: 148-58.

[13] Schenk S. MDMA self-administration in laboratory animals: a summary of the literature and proposal for future research. Neuropsychobiology 2009; 60: $130-6$.

[14] Heffernan TM, Ling J, Scholey AB. Subjective ratings of prospective memory deficits in MDMA ('Ecstasy') users. Hum Psychopharmacol 2001; 16: 33944.

[15] Green AR, Mechan AO, Elliott JM, O'Shea E, Colado MI. The pharmacology and clinical pharmacology of 3, 4-methylenedioxymethamphetamine (MDMA, "Ecstasy"). Pharmacol Rev 2003; 55: 463-508

[16] Broadbear JH, Tunstall B, Beringer K. Examining the role of oxytocin in the interoceptive effects of 3, 4-methylenedioxy-methamphetamine (MDMA, "Ecstasy") using a drug discrimination paradigm in the rat. Addict Biol 2010; doi: 10.1111/j.1369-1600.2010.00267.x.

[17] Morley KC, McGregor IS. (+/-) -3, 4-methylenedioxymethamphetamine (MDMA, 'Ecstasy') increases social interaction in rats. Eur J Pharmacol 2000; 408: 41-9.

\section{Jillian Broadbear (Guest Editor)}

School of Psychology and Psychiatry

Monash University

Australia

E-mail: jillian.broadbear@monash.edu
Andy Parrott

(Guest Editor)

Department of Psychology

Swansea University

UK

E-mail: a.c.parrott@swansea.ac.uk

(C) Broadbear and Parrott; Licensee Bentham Open.

This is an open access article licensed under the terms of the Creative Commons Attribution Non-Commercial License (http://creativecommons.org/licenses/ by$\mathrm{nc} / 3.0 /$ ) which permits unrestricted, non-commercial use, distribution and reproduction in any medium, provided the work is properly cited. 